\title{
Examining the Effectiveness of a Distant Learning Education Program: Use of Patient Safety and Reporting Law and Ethics as Example
}

\author{
Chiou-Fen Lin ${ }^{1}$, Meei-Ling Shyu ${ }^{1}$, Meei-Shiow Lu ${ }^{2}$, and Chung-I Huang ${ }^{3,4}$ \\ ${ }^{1}$ School of Nursing, Taipei Medical University \\ 2 The National Union of Nurses' Associations ROC \\ ${ }^{3}$ School of Health Care Administration, Taipei Medical University \\ ${ }^{4}$ Department of Technology Application and Human Resource Development, \\ National Taiwan Normal University, Taiwan
}

\begin{abstract}
This study was to test a distant learning education program aiming to improve nurses' understanding of law and ethics regarding to patient safety and reporting in Taiwan. With stratified randomized sampling of 3 medical centers, 12 regional and 50 local hospitals, 2,323 questionnaires were distributed to nurses working at the sampled hospitals. Questionnaire return rate was $99.5 \%$ with 2,312 valid returned. Based on this survey, the researchers designed a distant learning education course targeting the knowledge deficits. The results indicated that $57.7 \%$ of the sampled nurses passed the patient safety and reporting law and ethic test before the education program was instituted by the National Union of Nurses Associations. 38,603 nurses completed the distant learning course and $94.8 \%$ passed the test. This study demonstrated that the distant learning education program is effective, and should be considered as a way to improve access to and reduce cost of continuing education.
\end{abstract}

Keywords: Distant Learning Education Program, Patient Safety, Reporting, Law, Ethics.

\section{Introduction}

According to the Taiwanese Nursing Professional law no. 8, nurses should apply their practice license at the governing institute where the nurses practice. After register and receiving the practice license, the nurses can start nursing practice. The practicing nurses should receive certain hours of continuing education within 6 years in order to review the practice license (National Regulation Database, 2011). Therefore, all nursing associations have been providing all kinds of seminars and conferences to promote the accessibility of continuing nursing education. However, due to the time limitation and location issues, many nurses may not be able to participate in continuing education courses. Thus there is a need to explore the feasibility and effectiveness of distant learning education programs. 


\section{Study Purposes}

The purposes of this study were:

1. To assess nurses' knowledge of patient safety and reporting law and ethics.

2. To design the content of the distant learning education course on patient safety and reporting law and ethics.

3. To examine the effectiveness of the distant learning education course on patient safety and reporting law and ethics.

\section{Study Methods}

Multiple methods were used in this study, which included systematic review of literature, cross-sectional survey, distant learning education and outcome evaluation. This study comprised of three stages in implementation. The first stage was to appraise current literature. According to the study purposes, we searched and reviewed domestic and international nursing literature related to patient safety and reporting law and ethics. Based on the review findings, we constructed a draft of assessment tool, Knowledge Assessment of Patient Safety and Reporting Law and Ethics, to assess nurses' understanding of the topic. Expert validity and internal consistency were assessed. A pilot study was conducted with randomized sampling. Data were collected and analyzed to identify the final items of the assessment tool. The second stage was to conduct the knowledge assessment using the finalized tool. Based on the assessment results, we designed a distant learning education course specifically targeting the knowledge deficit. The third stage was to publish the distant learning education course content in the Newsletter of The National Unions of Nurses' Association, R.O.C. A post-test assessment was included. After studying the distant learning education course regarding to patient safety and reporting law and ethics, nurses completed the post-test assessment and faxed it back to The National Unions of Nurses' Association. Continuing education credits were granted to those passed the course evaluation.

\section{Study Population and Sampling Method}

The study population for assessing the knowledge of patient safety and reporting law and ethics was the domestic nurses working at the local or regional hospitals or medical centers (including those qualified based on the New Hospital Accreditation) for at least 6 months. Stratified random sampling method was used to select the hospitals from three different levels: medical centers (including those judged to be outstanding hospital based on the accreditation), regional hospitals (including those judged to be excellent hospital based on the accreditation), and local hospitals (including those judged to be qualified hospital based on the accreditation), according to the 2007-2009 hospital and teaching hospital accreditation results published by the Department of Health. Sample hospitals were selected from each of the three clusters to ensure the 
appropriate sample size in each cluster. Sample size was calculated based on the rule of having 10-20 samples in each concept or variable. Considering the size of the nursing population, we applied the large population sampling principle (Chiou, 2007) and estimated $1 \%$ attrition rate. This study planned to select about $11 \%$ of the nurses in Taiwan. We randomly selected three medical centers from 19 qualified medical centers, 12 regional hospitals from 92 regional hospitals, and 50 local hospitals from 432 local hospitals. The final sample included the day shift nurses working at one of these 65 hospitals for at least 6 months.

\section{$5 \quad$ Study Materials}

The demographic information was collected from the nurse participants, which included hospital, unit, professional level, years of working experience, age, gender, and educational level. The assessment tool, Knowledge Assessment of law and Ethics Regarding to Patient Safety and Reporting, included contents and performance of nurses' execution of patient safety reporting, knowledge of relevant law and ethics such as the purposes, benefits, types, ethics, and laws of reporting adverse events. A score was assigned to each item: the right answer received score 1 and the wrong answer score 0 . The total scores indicate the nurse's assessment score; the higher score the better knowledge on the patient safety and reporting laws and ethics. The final tool comprised of 16 multiple choice questions. To pass the assessment, nurses should correctly answer at least 12 questions (70\%).

1. Validity Test: Content validity of the assessment tool was evaluated by 10 experts of the patient safety and reporting system, from industry, regulation, and academics. The importance and suitability of each item were assessed. The content validity indexes were 0.8 and above. We also invited 5 nurses to assess the readability and wording. Revision was done to ensure the readability of the tool.

2. Reliability Test: Reliability was evaluated using the completed knowledge assessment tool from 92 nurses of a northern regional hospital. The results indicated that the internal consistency of the items, using Cronbach's $\alpha$ to express, ranged from 0.65 to 0.74 .

3. Discriminant Validity Test: The tool, Knowledge Assessment of Patient Safety and Reporting Law and Ethics, was further assessed for discriminate power. We grouped the nurses with the highest $27 \%$ total scores into the high performance group, and the nurses with the lowest $27 \%$ total scores in the low performance group. The different score in each item between the high performance and low performance groups formed the discriminant index. The original tool contained 20 items. We removed 4 items with discriminant index of 0.2 or below. The final tool contains a total of 16 items.

\section{Data Analysis and Statistics}

Knowledge Assessment of Patient Safety and Reporting Law and Ethics. Based on the stratified randomization, we obtained the consents from the sample hospitals for 
conducting research. During unit/ward morning meeting, a research staff explained the study purposes and invited the day shift nurses to participate in this study. After consenting, the nurse participants were given the knowledge assessment tool with name removed. A written instruction was given to the participating nurses for how to complete the study. The knowledge assessment tool was returned and examined for completeness upon return. A total of 2323 questionnaires were sent out with 2312 valid return. The valid return rate was $99.5 \%$. Data were entered into a computer software database and then analyzed using the Statistic Package for Social Study (SPSS, version 15.0). The nurse participants' demographic information were analyzed with descriptive statistics, including frequency, percentage, mean, and standard deviation. T-test, one way Analysis of variance (ANOVA) with Scheffe's posteriori comparison, and Pearson's correlation were used as inference statistics.

Evaluation of the Distant Learning Education Program. For each of the 16 items, it would be scored as 1 if the nurse's answer was correct and 0 if the answer was wrong. The total possible score was 16 if the nurse answered all items correctly. The pass score was set as $12(70 \%)$.

\section{$7 \quad$ Study Results}

Prior to conducting the distant learning education, there were only $57.7 \%$ of the participating nurses passed the knowledge assessment of patient safety and reporting law and ethics. The most frequent score was $12(18.4 \%)$, and the next frequent score was 11 and $13(16.3 \%)$. The mean score was 11.67 with standard deviation of 2.44 . There were 364 nurses, $15.8 \%$ of the nurse participants, received score of 10 or below. Nurses' knowledge on patient safety and reporting law and ethics was lower than their knowledge on patient safety. Among the 16 multiple choice questions, the majority of the nurses $(62.8 \%)$ answered wrong on the question, the reporting type for delaying medication administration due to computer out of order. The next common mistaken question was what kind of ethical conduct would be if the nurse helps the patient and his/her family to communicate with other medical professionals about the safety issues they concerned. About $57.1 \%$ of the participating nurses answered the question wrong. The results demonstrated that among the nurses'knowledge of patient safety and reporting law and ethics, there was particular needs to improve nurses' understandings of adverse event reporting type and ethical principles and rules. $\mathrm{cm}$

The distant learning education course was distributed through the Newsletters of the National Union of Nurses' Association. There were 38603 nurses read the distant learning education content and $94.8 \%$ of the participating nurses passed the knowledge assessment.

\section{Conclusions and Recommendations}

This study applied systematic review of literature, collected and compiled the relevant references on patient safety and reporting law and ethics. A cross-sectional survey was 
used to broadly and swiftly assess the nurses' knowledge related to patient safety and reporting law and ethics. Data analysis indicated that only $57.7 \%$ of the nurse participants passed the assessment. There was a need to improve nurse' knowledge on this topic. After implementing the distant learning education, the nurses increased their knowledge on patient safety and reporting law and ethics. There were 38603 nurses participated in the distant learning program and $94.8 \%$ passed the knowledge assessment. The nurse participants received immediate feedback from the distant learning education course and obtained the continuing education credits as a second gain. This study demonstrated the effectiveness of a distant learning education program on increasing nurses' knowledge of patient safety and reporting law and ethics. We recommended applying distant learning education programs in the future for national education topics, in order to improve the accessibility of continuing education and reduce the cost of delivering of the education.

Acknowledgement. This study was supported by the research fund from the Department of Health (DOH099-TD-M -113-099003). We thank the research team members for their active participation and the experts for taking time to attend the meetings. We also would like to express our gratitude to those nurses from the three medical centers, 12 regional hospitals, and 50 local hospitals and who completed the study assessment tool.

\section{References}

1. Chiou, H.J.: Quantitative research method (1): study design and data processing, Yeh Yeh, Taipei (2007) (in Chinese)

2. Nurse Act, Laws and Regulations Database of The Republic of China, Taiwan (2012), http://law.moj.gov.tw/LawClass/LawAll.aspx?PCode=L0020166 (in Chinese) 